\title{
Effect of Lanthanum Substrates on the Structural, Optical and Electrical Properties of Copper Selenide Thin Films Designed for 5G Technologies
}

\author{
Atef Qasrawi ( $\square$ atef.qasrawi@aaup.edu ) \\ İstinye Üniversitesi: Istinye Universitesi https://orcid.org/0000-0001-8193-6975 \\ Arwa N. Abu Ghannam \\ Arab American University
}

\section{Research Article}

Keywords: La/CuSe interfaces, thin film transistor, optical conductions, microwave resonator

Posted Date: September 28th, 2021

DOl: https://doi.org/10.21203/rs.3.rs-888845/v1

License: (c) (i) This work is licensed under a Creative Commons Attribution 4.0 International License.

Read Full License

Version of Record: A version of this preprint was published at Optical and Quantum Electronics on November 24th, 2021. See the published version at https://doi.org/10.1007/s11082-021-03375-z. 


\title{
Effect of lanthanum substrates on the structural, Optical and electrical
} properties of copper selenide thin films designed for $5 \mathrm{G}$ technologies

\section{A. F. Qasrawi ${ }^{1,2, *}$, Arwa N. Abu Ghannam ${ }^{1}$}

${ }^{1}$ Department of Physics, Arab American University, Jenin, Palestine

${ }^{2}$ Department of Electrical and Electronics Engineering, Istinye University, 34010, Istanbul, Turkey E-mail: atef.qasrawi@aaup.edu; atef.qasrawi@istinye.edu.tr

\begin{abstract}
In this work, copper selenide thin films coated onto glass and transparent lanthanum substrates are studied. The (glass, La)/CuSe thin films which are prepared by the thermal evaporation technique under a vacuum pressure of $10^{-5}$ mbar are structurally, morphologically, optically, dielectrically and electrically characterized. Lanthanum substrates improved the crystallinity by increasing the crystallite size and decreasing both of the microstrains and defect density of copper selenide. La substrates redshifts the energy band gap and doubled the dielectric constant values. In addition, employing Drude-Lorentz approaches for optical conduction to fit the dielectric constant provided information about the effects of La substrates on the drift mobility, plasmon frequency, free carrier density and scattering times at femtosecond level. The drift mobility increased and the plasmon frequency range is modified when La substrates are used. Verifying impedance spectroscopy test in the microwave frequency domain have shown that the $\mathrm{La}($ gate)/CuSe/Ag (source) transistors can be employed as band pass filter suitable for $5 \mathrm{G}$ technologies. The microwave cutoff frequency reached $\sim 5.0 \mathrm{GHz}$ at a notch frequency of $2.80 \mathrm{GHz}$ of the $\mathrm{La} / \mathrm{CuSe} / \mathrm{Ag}$ highpass filters.
\end{abstract}

Keywords: La/CuSe interfaces; thin film transistor; optical conductions; microwave resonator

* Author to whom any correspondence should be addressed. 


\section{Introduction}

Copper selenide thin films have captured wide interest due to their applications as electrocatalysts, counter electrodes in solar cells and self-repairable electrodes [1]. They show novel thermoelectric properties and mentioned being suitable for flexible electronics [1]. CuSe films also find its location in other class of applications including photocatalysis and thermal phototherapy [2]. In addition, studies which care about plasmonic interactions in copper selenide have shown their suitability for use in biomedical applications [3].

Various preparation methods including physical vapor deposition techniques have been employed to improve the performance of coper selenide thin films $[1,5]$. Each preparation method has it is own properties. As for examples, CuSe thin films prepared by the chemical bath deposition technique was not adherents with the substrate at higher precursor concentration [1]. Films prepared by the electrodeposition technique provided an adherent and uniform films on the substrates but flexible substrate (polymeric) cannot be used in this method. On the other hand, the substrate used for deposition of CuSe films play an additional role on justifying the physical properties of the films. When polyvinyl chloride, polyvinyl alcohol, and paper is employed as substrates to grow $\mathrm{CuSe}$ films, the resulting films are found to be highly conductive. The achieved high conductivities remained for several months. The properties of these films were not affected by stretching, bending and folding [1].

The applied preparation methods on various types of substrates for depositing copper selenide films motivated us to prepare copper thin films by the thermal evaporation technique onto conductive transparent rare earth lanthanum substrates. The thermal evaporation technique is employed because of its relative simplicity, low cost of the apparatus, higher deposition rates, and scalability [5]. Lanthanum is selected because it is highly transparent material in the spectral range of 4.0-1.0 eV, exhibiting the electronic shell configuration of $5 \mathrm{~d}^{1} 6 \mathrm{~s}^{2}$ [6]. The orbital states of lanthanum can reach those of $\mathrm{CuSe}$ allowing easier electronic conduction and optical transitions. For this reason, here in this work, we will report the structural, morphological, optical, 
dielectric and optical properties of copper selenide thin films coated onto glass and transparent lanthanum substrates of thickness of $150 \mathrm{~nm}$. The effects of the La substrates on the lattice parameters, crystallite size, microstrain, stacking faults, optical transmittance, reflectance, absorption coefficient, energy band gap, dielectric constant and on the microwave band pass characteristics are reported.

\section{Experimental details}

Transparent lanthanum substrates of thicknesses of $150 \mathrm{~nm}$ were coated onto ultrasonically cleaned glass substrates in a NORM VCM-600 physical vapor deposition system. The vacuum pressure was kept at $\sim 10^{-5}$ mbar. The source material was lanthanum powders (99.99\%, Alpha Aesar). The lanthanum substrates were then covered with a1.0 $\mu$ m thick copper selenide (99.99\%, Alpha Aesar ) using NORM VCM-600 physical vapor deposition system. Some of the CuSe samples were produced onto glass without lanthanum. La/CuSe films were masked with rectangular masks of areas of 0.0314 $\mathrm{cm}^{2}$ to produce $\mathrm{Ag}$ metal pads on the top surface of CuSe layer. For the purpose of more accurate electrical measurements the thickness of some of the La substrates was raised to $300-350 \mathrm{~nm}$. The thickness of the La, glass/CuSe and $\mathrm{La} / \mathrm{CuSe}$ films were measured with the help of an in situ monitoring Inficon STM-2 thickness monitors. The X-ray diffraction patterns were recorded using MiniFlex $600 \mathrm{X}$-ray diffraction unit at a scanning speed of $0.5^{\circ} /$ minute. The optical transmittance

and reflectance spectra were measured with the help of Thermoscientific Evolution 300 spectrophotometer. The impedance spectra were recorded with the help of Agilent 4291B 1.0 M$1.8 \mathrm{GHz}$ impedance analyzer.

\section{Results and discussion}

Thin films of copper selenide coated onto glass and $150 \mathrm{~nm}$ thick transparent lanthanum substrates are shown in inset-1 of Fig. 1. It is clear from the images that the color of CuSe thin films has changed from dark brown to light brown. The change in the color gives evidence about the possible changes in optical properties. Fig. 1 also displays the recorded X-ray diffraction (XRD) patterns for copper 
selenide films in the presence and absence of lanthanum substrates. The XRD patterns which are analyzed with the help of "TREOR 92" software packages revealed cubic structure of CuSe. All the observed peaks are indexed for the cubic phase of CuSe (PDF card No: 01-071-4325). When La replaces glass, the calculated lattice constant of the cubic CuSe $(a=5.697 \AA)$ decreases to $a=$ $5.656 \AA$. XRD patterns of (glass, La)/CuSe contained a peak centered at $2 \theta=24.45^{\circ}$ which are related to the orthorhombic phase of $\mathrm{CuSe}$ ((PDF card no: 2027-0184). In addition, The XRD patterns of CuSe coated onto lanthanum substrates displayed some peaks of hexagonal lanthanum $(a=$ $3.772 \AA$ and $c=12.141 \AA[6])$

The lattice mismatches, $\Delta_{a} \%=\frac{\left|a_{L a}-a_{C u S e}\right|}{a_{C u S e}} \times 100 \%$, between the hexagonal lanthanum substrates and cubic CuSe along the $a$-and $c$-axis are $33.3 \%$ and $114.6 \%$, respectively. Large lattice mismatches are mentioned resulting in interfacial stresses and forms three dimensional quantum confinements [7, 8]. Three-dimensional quantum confinement is mentioned capable of altering radically the nonlinear optical properties of semiconductors in the transparency regions [9]. On the other hand, as can be seen from inset-2 of Fig. 1, the maximum peak of the observed XRD patterns of CuSe shifts toward larger angles. The shift is associated with a decrease in the maximum peak intensity. This behavior which should have resulted from the large lattice mismatches between the two stacked layers ( $\mathrm{La}$ and $\mathrm{CuSe}$ ) is an indication of lattice modification due to lanthanum incorporation. Particularly, the structural parameters [10] presented by the crystallite size $\left(D=\frac{0.94 \lambda}{\beta \cos \theta} ; \beta\right.$ : maximum peak broadening), strain $\left(\varepsilon=\frac{\beta}{4 \tan \theta}\right)$, staking faults $\left(S F \%=\frac{2 \pi^{2} \beta}{45 \sqrt{3 \tan (\theta)}}\right)$, dislocation density $\left(\delta=\frac{15 \varepsilon}{a D}\right)$ and stress $(S=\gamma \epsilon ; \gamma=62 \mathrm{GPa}$ [11]) which are calculated and listed in Table-I provide evidences about the structural modifications. As seen from the table, the crystallite size increased by $14.2 \%$ while the microstrain, the stress, the stacking faults and the defect density decreased by $14.0 \%, 14.0 \%, 14.8 \%, 24.9 \%$, respectively. The changes in the structural parameters confirm the enhanced crystallinity due to La incorporation. Physically, the ionic radius $\left(r_{L a}\right)$ of $\mathrm{La}^{+3}$ being $1.16 \AA$ [12] cannot substitute copper $\left(\mathrm{Cu}^{+2} ; r_{C u}=72 \mathrm{pm} \mathrm{[13])} \mathrm{vacancy.} \mathrm{Instead,} \mathrm{it} \mathrm{can} \mathrm{make}\right.$ 
atomic interstitial substitutions. Thus, the defect density should have increased [12]. However, as the La-La bond length is (3.918 $\AA$ [14]) larger than that of La-Se (3.123 $\AA$ [15]) by $25.4 \%$, broken bonds of lanthanum prefer bonding with excess Selenium atoms. It is reported that nanocrystals also have high surface energy due to the incomplete bonding at the surface. To accommodate the high surface energy, nanocrystals tend to aggregate [16]. Reduction of surface energy is believed to be the driving factor for grain (accumulation of crystallites) growth [17].

The effect of the lanthanum substrates on the stoichiometric composition and surface morphology of CuSe films can be read from Fig. 2. The figure show large area of $\mathrm{La} / \mathrm{CuSe}$ films being selected for energy dispersive X-ray spectroscopic (EDS) analysis. Over many selected regions of different samples being prepared in the same and/or sequential growth cycles, one may see that glass/CuSe (inset-1 of Fig. 2) and La/CuSe films exhibit variations in the compositional stoichiometry. Namely, while films coated onto glass substrates have tendencies to form wire like $\left(1200 \times 125(\mathrm{~nm})^{2}\right)$ $\mathrm{Cu}_{0.86} \mathrm{Se}$ grains impeded in an amorphous sea of $\mathrm{Cu}_{1.19} \mathrm{Se}$, those coated onto La substrates prefer forming rectangular grains of sizes of $382 \mathrm{~nm}$. Above each rectangular grain, a tiny grain of size of $\sim 105 \mathrm{~nm}$ (while colored grains in inset-2 of Fig. 2) can be observed. The EDS test for the rectangular grains show predicts the formation of $\mathrm{Cu}_{1.68} \mathrm{Se}$. The white colored tiny grains mostly composed of $\mathrm{Cu}_{1.73-2.10} \mathrm{Se}$. In other words, lanthanum induced the formation of $\mathrm{Cu}$ deficient $\mathrm{Cu}_{2} \mathrm{Se}$. Although films coated onto glass substrates prefer the formation of $\mathrm{CuSe}$, those coated onto lanthanum prefer formation of deficient $\mathrm{Cu}_{2} \mathrm{Se}$. Recent works which targeted studying the atomic mechanism of ionic confinement in copper selenide have shown that $\mathrm{Cu}_{2} \mathrm{Se}$ tend to retain non-fast ionic conducting phase in the grain boundaries in the presence of bismuth as interstitial doping agent. It is reported that under conditions where the interstitial doping agent blocks the migration of $\mathrm{Cu}$ ions, barriers inside the $\mathrm{Cu}_{2} \mathrm{Se}$ grains and interfacial phases in the boundaries of $\mathrm{Cu}_{2} \mathrm{Se}$ effectively split a large $\mathrm{Cu}_{2} \mathrm{Se}$ grain into a number of small domains leading to the loss of $\mathrm{Cu}$ [18]. It is also stated that the atomic-scale doping in the core of the defects stabilizes the defects and behave as barriers inside the $\mathrm{Cu}_{2} \mathrm{Se}_{\text {grain }}$. 
Hence, the atomic-scale doping prompts the formation of the defective interfacial phase in the grain boundaries [18] as we also observed from the XRD analyses.

The effect of La substrates on the optical transmittance $(T)$, reflectance $(R)$ and absorption coefficient ( $\alpha$ ) spectra is evident from Fig. 3 (a), (b) and (c), respectively. As Fig.3 (a) illustrates, the sharp transmission is initiated as the incident photon energy $(E)$ become lower than $2.60 \mathrm{eV} . T$ spectra of glass $/ \mathrm{CuSe}$ exhibit maxima of $32 \%$ at $E=1.82 \mathrm{eV}$. The transmittance spectra redshifts exhibiting maxima $27 \%$ at $1.68 \mathrm{eV}$ as La replace glass. In addition, the reflectively of glass/CuSe films is relatively low $\left(R_{\max }<4 \%\right)$. Coating of CuSe onto La substrates increase $R$ values in all the studied range of light. The maximum reflectance value being $11 \%$ is observed at $E=1.14 \mathrm{eV}$. On the other hand, the absorption coefficient (Fig. 3 (b)) for films of thicknesses of $t$ is calculated with the help of the equation [19],

$$
T=\frac{\left(1-R_{\text {glass }}\right)\left(1-R_{L a}\right)\left(1-R_{\text {La }} \text { CuSe }\right) e^{-\alpha t}}{1-R_{\text {glass }} R_{L a} R_{L a / C u S e} e^{-3 \alpha t}} .
$$

As can be seen from Fig. 3 (b), copper selenide thin films coated onto glass substrates exhibited strong absorption in the incident photon energy ranges of 4.0-3.15 eV and 2.80-2.10 eV. In the range of 3.15 $-2.80 \mathrm{eV}, \alpha$ spectra of glass/CuSe display absorption saturation. The absorption saturation usually originates from band filling and carrier screening by the excited carriers [20]. Replacement of glass by lanthanum substrates extended the absorption saturation range (4.0-2.6 eV) and forces disappearance of strong absorption in the high energy range (4.0-3.15 eV). In the low energy range (1.9-1.1 eV), both of the glass/CuSe and $\mathrm{La} / \mathrm{CuSe}$ displayed increased values of $\alpha$ with decreasing incident photon energy. The increase in the absorption coefficient values with decreasing incident photon energies is ascribed to the free carrier absorption in the films. Free carrier absorption phenomena were also observed for $\mathrm{La} / \mathrm{Cd}_{2} \mathrm{~S}_{3}$ thin films [20]. The phenomena are assigned to the ability of long wavelengths (IR range) to reach the lattice and motivates phonon excitations. Excited phonons set charge carriers free, allowing them to recombine with holes forming an additional polarized electron-hole pairs [20]. The nonstoichiometric composition of copper selenide (our EDS 
results) which result in high order degeneracy could also account for the free carrier absorption in CuSe [21].

To get information about the changes in the optical transitions that resulted from replacement of glass by lanthanum substrates, the energy band gap $\left(E_{g}\right)$ is calculated with the help of Tauc's equation $(\alpha E)^{2} \propto\left(E-E_{g}\right)[19,20]$. As illustrated in Fig. 3 (c), the linear plots of the $(\alpha E)^{2}-E$ variations crosses the $E$-axis at $E=E_{g}=2.30 \mathrm{eV}$ and $E=E_{g}=2.23 \mathrm{eV}$, for glass $/ \mathrm{CuSe}$ and La/CuSe films, respectively. The obtained energy band gap of glass/CuSe is consistent with literature data [22]. The redshift in the energy band gap is ascribed to the orbital over lapping. From the electronic configuration point of view, the electronic distribution of the $\mathrm{Cu}$ and Se atoms reach $3 \mathrm{~d}^{10} 4 \mathrm{~s}^{1}$ and $3 d^{10} 4 s^{2} 4 p^{4}$, respectively. On the other hand, the electronic configuration of La metal reaches $5 d^{1} 6 s^{2}$. The lanthanum's higher states make the overlapping of the La orbitals with that of $\mathrm{Cu}$ and Se strongly preferable. In addition, the Schottky nature of La/CuSe interfaces forces the flow of holes from $p$-type CuSe (verified by hot-probe technique) to La metal causing a band bending due to energy barrier height formation [23]. Another reason for the band gap shrinkage in the presence of metallic substrates is the image force lowering. The image charges lowers the potential barrier to the flow of holes leading to the up bending of the valance band [23].

The effect of Lanthanum substrates on the dielectric properties of copper selenide thin films is readable from Fig. 4 (a) and (b) respectively. The real $\left(\varepsilon_{r}\right)$ and imaginary $\left(\varepsilon_{i m}\right)$ parts of the dielectric constant are calculated from the equations [20],

$$
\begin{aligned}
& \varepsilon_{r}=\varepsilon_{e f f}-k^{2}, \\
& \varepsilon_{i m}=2 \sqrt{\varepsilon_{e f f}} k, \\
& R=\frac{\left(\sqrt{\varepsilon_{e f f}}-1\right)^{2}+k^{2}}{\left(\sqrt{\varepsilon_{e f f}}+1\right)^{2}+k^{2}} \\
& k=\frac{\alpha \lambda}{4 \pi} .
\end{aligned}
$$

In accordance with Fig. 4 (a), the real part of the dielectric constant of glass/CuSe display broaden peaks at critical energy values of 2.30 and $1.82 \mathrm{eV}$. Replacing glass by lanthanum substrates leads to 
the appearance of peaks at 2.59 and $1.99 \mathrm{eV}$. In addition, the value of $\varepsilon_{r}$ is increased by about two times. $\varepsilon_{r}-E$ variations also follow faster decaying trends in the IR range as a result of using lanthanum instead of glass. The critical energy values being $2.30 \mathrm{eV}$ observed in $\varepsilon_{r}$ spectra of glass/CuSe is assigned to the direct allowed transitions within the band gap of CuSe. The critical energy value centered at $1.82 \mathrm{eV}$ is assigned to transitions from the valence band maxima centered at $R_{3,4 v}$ of the first Brillouin zone of selenium to the conduction band minima centered at $H_{6 c}$ of the first Brillouin zone of Se [24], respectively. On the other hand, the strong interaction between La and Se seems to be dominant oscillator in the real part of the dielectric spectra. Namely, the critical energy value being $2.59 \mathrm{eV}$ which appeared in $\varepsilon_{r}$ spectra of $\mathrm{La} / \mathrm{CuSe}$ corresponds to the direct allowed transitions energy band gap of $\mathrm{La}_{2} \mathrm{Se}_{3}$ [25]. The critical energy centered at $1.99 \mathrm{eV}$ is also assigned to optical transitions in selenium [26].

Fig. 4 (b) display the imaginary part of the dielectric constant. The imaginary part is directly proportional with the optical conductivity $\left(\check{\sigma}(w)=\frac{\varepsilon_{i m} w}{4 \pi} ; w=2 \pi c / \lambda\right)$. As seen from the figure, except for the ultraviolet range of light, no remarkable change in the value of the imaginary part can be detected. In the spectral range of $4.0-3.16 \mathrm{eV}, \mathrm{La} / \mathrm{CuSe}$ is less conductive than that of glass/CuSe. Slight enhancement in the imaginary part value can be observed in the IR range of light. Modeling the imaginary part of dielectric spectra in accordance with the Drude-Lorentz approach [19, 20] allowed determining the optical conductivity parameters. The dispersion relation of the imaginary part of dielectric constant takes the form,

$$
\varepsilon_{i m}=\sum_{i=1}^{k} \frac{w_{p e_{i}}^{2} w}{\tau_{i}\left(\left(w_{e_{i}}^{2}-w^{2}\right)^{2}+w^{2} \tau_{i}^{-2}\right)}
$$

In Eqn. (6), $w$ is the radial frequency of light signals, $w_{p e}=\sqrt{4 \pi P e^{2} / m^{*}}$ is the plasmon frequency, $W_{e i}$ is the charge carrier-plasmon coupled oscillator frequency, $\tau_{i}$ is the average scattering time at femtosecond level, $P$ is the free charge carrier density and $m^{*}=\left(m_{L a}^{*^{-1}}+m_{C u}^{*^{-1}} s e\right)^{-1}$ is the reduced effective mass of the $\mathrm{La} / \mathrm{CuSe}$ system. Inserting the values of $m_{C u}^{*} \quad s e=0.5 m_{o}[27]$ and $m_{L a}^{*}=1.0 m_{o}$ 
[20] for copper selenide and lanthanum, respectively, and executing the series up to $k=5$ is sufficient to reproduce the experimental data. The theoretically estimated dielectric constant is plotted by circles in Fig. 4 (b). The fitting of the $\varepsilon_{i m}$ spectra allowed determining the optical conductivity parameters which are shown in Table-II. As seen from the table, for the IR oscillator centered near $E_{e}=1.1 \mathrm{eV}$, the free hole concentration decreases, the plasmon frequency decreases and the drift mobility increases by replacing glass substrates with lanthanum. The enhanced mobility is probably due to the completed bonding of dangling bonds of Se with lanthanum at the interface. In addition the easier electronic motion originated from atomic overlapping could also account for the improved mobility values. The other oscillators in the visible range of light $(2.68 \mathrm{eV}$ and $2.89 \mathrm{eV})$ display similar characteristics. Oscillators dominant in the ultraviolet range of light $(3.93,4.14 \mathrm{eV})$ show no remarkable change in the optical conductivity parameters. The scattering time at femtosecond level which is the inverse of the damping coefficient of the electronic frictional forces [20] slightly increases for the first and second oscillators $\left(E_{e}=1.1 \mathrm{eV}\right.$ and $\left.2.68 \mathrm{eV}\right)$. The increase in this value means less resistance to the charge carrier motion. Hence drift mobility is increased.

One interesting feature which is worth of focusing, here, is the values of plasmon frequency. The plasmon frequency exhibit values in the range of $2.30-6.51 \mathrm{GHz}$. It indicates that signals of such frequencies can pass through $\mathrm{La} / \mathrm{CuSe}$ interfaces. Signals exhibiting values less than $2.30 \mathrm{GHz}$ are rejected. To verify this belief we have tested the samples through constructing a $\mathrm{La}$ (gate)/CuSe/Ag (drain, source) transistor channels. An ac signal of low amplitude is imposed between the terminals of source (Ag) and gate (La). The resulting ac conductivity $(\sigma)$, impedance $(Z)$ and magnitude of reflection coefficient $\left(S_{11} ; Z_{L a / C u 2 S e}=\frac{S_{11}+1}{S_{11}-1} Z_{\text {source }}\right.$ [28]) spectra are shown in Fig. 5 (a), (b) and (c), respectively. It is clear from Fig. 5 (a) that the higher the signal frequency, the more conductive the channel. The conductivity increases from $0.06(\Omega \mathrm{cm})^{-1}$ to $\sim 140(\Omega \mathrm{cm})^{-1}$ as the signal frequency increases from 0.01 to $1.80 \mathrm{GHz}$. It means that the closer the signal frequency to the plasmon range, the easier the pass of the electrical signal. Opposite behavior can be observed from the impedance spectra. The larger the ac signal frequency the less the impedance. The $Z-f$ variation is linear in 
most of the studied range. On the other hand, as $S_{11}$ spectra show, the larger the frequency, the less the value of $S_{11}$. This parameter provides information about the input-output relationship between ports at terminals of antenna system. $S_{11}=0.0$ means that all incident signals are fully transmitted with no wave power loss. $S_{11}=1.0$ indicates that all incident electromagnetic signals are rejected. Hence, $S_{11}$ spectra which are illustrated in Fig. 5 (c) represent a band pass filter characteristics with notch frequency predictable from modeling the transistors as series resistance (R) inductance (L) - capacitnace (C) $(R L C)$ circuit. For this circuit, $Z_{L a / C u S e / A g}=R+j w L-j w C$ $[28,29]$. The experimental data is reproduced (blue colored circles) by substituting $R=100 \Omega$, $L=10 \mathrm{nH}$ and $C=0.45 \mathrm{pF}$. These parameters result in a microwave cutoff frequency $f_{c o}=$ $(2 \pi R C)^{-1}$ of $3.54 \mathrm{GHz}$. The practically determined cutoff frequency is within the range of plasmon frequency identified from the optical measurements. The ideal parameters (black circles in Fig. 5 (c)) of the La/CuSe/Ag band filters are $R=50 \Omega, L=5 \mathrm{nH}$ and $C=0.64 \mathrm{pF}$ and $f_{c o}=4.98 \mathrm{GHz}$. The notch frequency $\left(f_{n}\right)$ of the filter is $2.8 \mathrm{GHz}$. The numerical data indicate the possible application of the $\mathrm{La} / \mathrm{CuSe} / \mathrm{Ag}$ transistors from filtering $5 \mathrm{G}$ signals. 5G technology require resonators workability in the frequency domain of 3.6-26 GHz for 5G technology [29-31].

\section{Conclusions}

Herein, the effect of transparent lanthanum substrates on the structural, optical, dielectric and electrical properties of copper selenide thin films are investigated. Thin films of copper selenide are observed to exhibit enhanced crystallinity accompanied with redshift in the energy band gap and larger dielectric constant values as a result of replacing glass by lanthanum substrates. In addition, the coating of copper selenide onto lanthanum substrates increased the drift mobility values and decreased the friction to charge transport in the films. Verifying electrical tests has shown the suitability of the $\mathrm{La} / \mathrm{CuSe} / \mathrm{Ag}$ transistors to behave as band filters which allow signals pass in the gigahertz level. Such property makes the currently proposed interface suitable for 5G mobile technology. 


\section{cknowledgements}

This work was supported by the Deanship of Scientific Research (DSR), Arab American University, Jenin, Palestine and by DSR of Istinye University, Istanbul, Turkey. The authors, therefore, gratefully acknowledge the DSR technical and financial support.

\section{Conflict of Interest}

The authors declare that they have no conflict of interest

\section{Funding:}

This study was funded by the Deanship of Scientific Research (DSR), Arab American University, Jenin, Palestine and by Istinye University, Istanbul, Turkey.

\section{Data Availability Statement}

The data that support the findings of this study are available from the corresponding author upon reasonable request.

\section{References}

\section{References}

[1] Hussain, R. A., Hussain, I.: Copper selenide thin films from growth to applications. Solid State Sci. 100, $106101(2020)$

[2] Wang, X., Miao, Z., Ma, Y., Chen, H., Qian, H., Zha, Z.: One-pot solution synthesis of shape-controlled copper selenide nanostructures and their potential applications in photocatalysis and photothermal therapy. Nanoscale 9(38), 14512-14519 (2017)

[3] Ai, K., Huang, J., Xiao, Z., Yang, Y., Bai, Y., Peng, J.: Localized surface plasmon resonance properties and biomedical applications of copper selenide nanomaterials. Materials Today Chemistry 20, 100402 (2021)

[4] Malavekar, D. B., Bulakhe, R. N., Kale, S. B., Patil, U. M., In, I., Lokhande, C. D.: Synthesis of layered copper selenide on reduced graphene oxide sheets via SILAR method for flexible asymmetric solidstate supercapacitor. J. Alloy. Compd. 869, 159198 (2021)

[5] Abd-Elnaiem, A. M., Mahmoud, A. Z., and Moustafa S.: Structural and optical properties of thermally evaporated and annealed Ge20Se76Sn4 thin films." Optical Materials 111110607 (2021).

[6] Polyakov, O.: Technology of Ferroalloys with Rare-Earth Metals. In Handbook of Ferroalloys (pp. 459469). Butterworth-Heinemann (2013) 
[7] Khusayfan, N. M., Khanfar, H. K., Alharbi, S. R.: Design and Characterization of Au/CdSe/GeO 2/C MOSFET Devices. Materials Research 24, (2021)

[8] Algarni, S. E., Qasrawi, A. F., Khusayfan, N. M.: Design and characterization of ZnSe/GeO2 heterojunctions as bandstop filters and negative capacitance devices. physica status solidi (a) 218(8), $2000830(2021)$

[9] Cotter, D., Burt, M. G., Manning, R. J.: Below-band-gap third-order optical nonlinearity of nanometer-size semiconductor crystallites. Phys. Rev. Let. 68(8), 1200 (1992)

[10] AIGarni, S. E., Qasrawi, A. F.: Indium slabs induced structural phase transitions and their effects on the electrical and optical properties of stacked layers of the thermally annealed Cu2O thin films. Results Phys. 16, 102901 (2020)

[11] Namsani, S., Gahtori, B., Auluck, S., Singh, J. K.: An interaction potential to study the thermal structure evolution of a thermoelectric material: $\beta$-Cu2Se. J. Comput. Chem. 38(25), 2161-2170 (2017)

[12] Lee, W., Chen, S. Y., Tseng, E., Gloter, A., Chen, C. L.: Study of defect structure in ferromagnetic nanocrystalline CeO2: effect of ionic radius. The Journal of Physical Chemistry C 120(27), 14874-14882 (2016)

[13] Tian, C., Cheng, J., Yang, J.: A highly active cathode material of Cu-doped Sr 2 Fe 1.5 Mo $0.5 \mathrm{O} 6$ for symmetrical solid oxide fuel cells. Journal of Materials Science: Materials in Electronics 32(1), 1258$1264(2021)$

[14] Arumugam, S., Saravanan, C., Thiyagarajan, R., Rao, G. N.: Effect of hydrostatic pressure on electrical resistivity of La0. $5 \mathrm{Ca} 0.5 \mathrm{Mn} 1-\mathrm{xMoxO}(\mathrm{x}=0.03$ and 0.05$)$ manganites: Experimental and theoretical approaches. J. Magn. Magn. Mater. 507, 166775 (2020)

[15] Guschlbauer, J., Vollgraff, T., Xie, X., Fetoh, A., Sundermeyer, J.: Heavy Silylchalcogenido Lanthanates Ph4P [Cp3La-ESiMe3](E=S, Se, Te) via Fluoride-Induced Demethylation of Dimethylcarbonate to Ph4P [OCO2Me] Key Intermediate. Dalton T. (2021) 10.1039/d1dt02000e

[16] Bruck, A. M., Cama, C. A., Gannett, C. N., Marschilok, A. C., Takeuchi, E. S., Takeuchi, K. J.: Nanocrystalline iron oxide based electroactive materials in lithium ion batteries: the critical role of crystallite size, morphology, and electrode heterostructure on battery relevant electrochemistry. Inorg. Chem. Front. 3(1), 26-40 (2016)

[17] Janney, M. A., Kimrey, H. D., Schmidt, M. A., Kiggans, J. O.: Grain growth in microwave-annealed alumina. J. Am. Ceram. Soc. 74(7), 1675-1681 (1991) 
[18] Yang, D., Bai, H., Su, X., Li, Z., Luo, T., Li, J., Tang, X.: Atomic mechanism of ionic confinement in the thermoelectric Cu2Se based on a low-cost electric-current method. Cell Reports Physical Science 2(2), $100345(2021)$

[19] Pankove, J. I.: Optical processes in semiconductors. Courier Corporation (1975)

[20] Qasrawi, A. F., Omareya, O. A.: Formation and Characterization of Cd 2 S 3 Polycrystalline Films onto Glass and Lanthanum Substrates. J. Electron. Mater. 48(4), 2350-2355 (2019)

[21] Chakrabarti, D. J., Laughlin, D. E.: The Cu- Se (copper-selenium) system. Bulletin of alloy phase diagrams 2(3), 305-315 (1981)

[22] Barman, B., Handique, K. C., Nanung, Y., Kalita, P. K.: Synthesis and characterization of chemically synthesized CuSe nanoparticles for photovoltaic application. Materials Today: Proceedings 46, 6213$6217(2021)$

[23] Sze, S. M., Li, Y., Ng, K. K.: Physics of semiconductor devices. John wiley \& sons (2021)

Råsander, M., Bergqvist, L., Delin, A.: Density functional theory study of the electronic structure of fluorite Cu2Se. Journal of Physics: Condensed Matter 25(12), 125503 (2013)

[24] Bagde, G. D., Sartale, S. D., Lokhande, C. D.: Spray pyrolytic deposition and characterization of lanthanum selenide (La2Se3) thin films. App. Surf. Sci. 214(1-4), 27-35 (2003)

[25] Solieman, A., Abu-Sehly, A. A.: Modelling of optical properties of amorphous selenium thin films. Physica B: Condensed Matter 405(4), 1101-1107 (2010)

[26] Alharbi, S. R., Qasrawi, A. F.: Structural and optoelectronic properties of MoO3/CuSe interfaces. physica status solidi (a) 216(6), 1800977 (2019)

[27] Pozar, D. M.: Microwave engineering. John wiley \& sons (2011)

[28] Al-Yasir, Y. I., Ojaroudi Parchin, N., Abd-Alhameed, R. A., Abdulkhaleq, A. M., Noras, J. M.: Recent progress in the design of $4 \mathrm{G} / 5 \mathrm{G}$ reconfigurable filters. Electronics 8(1), 114 (2019)

[29] Al-Yasir, Y. I., Parchin, N. O., Alabdallah, A., Abdulkhaleq, A. M., Sajedin, M., Elfergani, I. T., AbdAlhameed, R. A.: Design, simulation and implementation of very compact open-loop trisection BPF for 5G communications. In 2019 IEEE 2nd 5G World Forum (5GWF) (pp. 189-193). IEEE (2019, September) 10.1109/5GWF.2019.8911677.

[30] Watanabe, A. O., Lin, T. H., Ali, M., Wang, Y., Smet, V., Raj, P. M., Swaminathan, M.: Ultrathin AntennaIntegrated Glass-Based Millimeter-Wave Package With Through-Glass Vias. IEEE T. Microw. Theory 68(12), 5082-5092 (2020) 
Table -I Structural parameters of glass/CuSe and $\mathrm{La} / \mathrm{CuSe}$ films.

\begin{tabular}{|c|c|c|c|c|c|c|}
\hline & $a(\mathrm{~A})$ & $\mathbf{D}(\mathbf{n m})$ & $\varepsilon\left(\times 10^{-3}\right)$ & $\mathrm{S}\left(\mathrm{x10}{ }^{9}\right.$ dyne $\left./ \mathrm{cm}^{2}\right)$ & SF\% & $\delta\left(\times 10^{11}\right.$ line $\left./ \mathrm{cm}^{2}\right)$ \\
\hline glass/CuSe & 5.697 & 28 & 5.43 & 3.37 & 0.27 & 5.02 \\
\hline $\mathrm{La} / \mathrm{CuSe}$ & 5.656 & 32 & 4.67 & 2.89 & 0.23 & 3.77 \\
\hline
\end{tabular}

Table-II: Optical conductivity parameters for (glass, La)/CuSe films

\begin{tabular}{|c|c|c|c|c|c|c|c|c|c|c|}
\hline & \multicolumn{5}{|c|}{ Glass/CuSe } & \multicolumn{5}{|c|}{$\mathrm{La} / \mathrm{CuSe}$} \\
\hline & 1 & 2 & 3 & 4 & 5 & 1 & 2 & 3 & 4 & 5 \\
\hline$\tau$ i (fs) & 1.50 & 1.00 & 0.50 & 0.45 & 0.30 & 1.55 & 1.40 & 0.50 & 0.30 & 0.10 \\
\hline $\begin{array}{c}\text { We }\left(\times 10^{15}\right. \\
\text { Rad./s })\end{array}$ & 1.70 & 4.10 & 4.40 & 6.00 & 6.30 & 1.62 & 4.00 & 4.40 & 6.00 & 6.00 \\
\hline$E_{e}(\mathrm{eV})$ & 1.11 & 2.68 & 2.89 & 3.93 & 4.14 & 1.06 & 2.62 & 2.89 & 3.93 & 3.93 \\
\hline$p\left(\begin{array}{c}\times 10^{18} \mathrm{~cm}^{-} \\
3)\end{array}\right.$ & 8.00 & 7.00 & 20.0 & 53.0 & 8.00 & 6.60 & 2.60 & 14.0 & 40.0 & 5.00 \\
\hline$\mu\left(\mathrm{cm}^{2} / \mathrm{Vs}\right)$ & 5.30 & 3.50 & 1.80 & 1.60 & 0.40 & 8.20 & 7.40 & 2.63 & 1.60 & 0.11 \\
\hline $\mathbf{w}_{\text {pi }}(\mathbf{G H z})$ & 2.38 & 2.22 & 3.76 & 6.12 & 2.38 & 2.64 & 1.66 & 3.85 & 6.51 & 2.30 \\
\hline
\end{tabular}




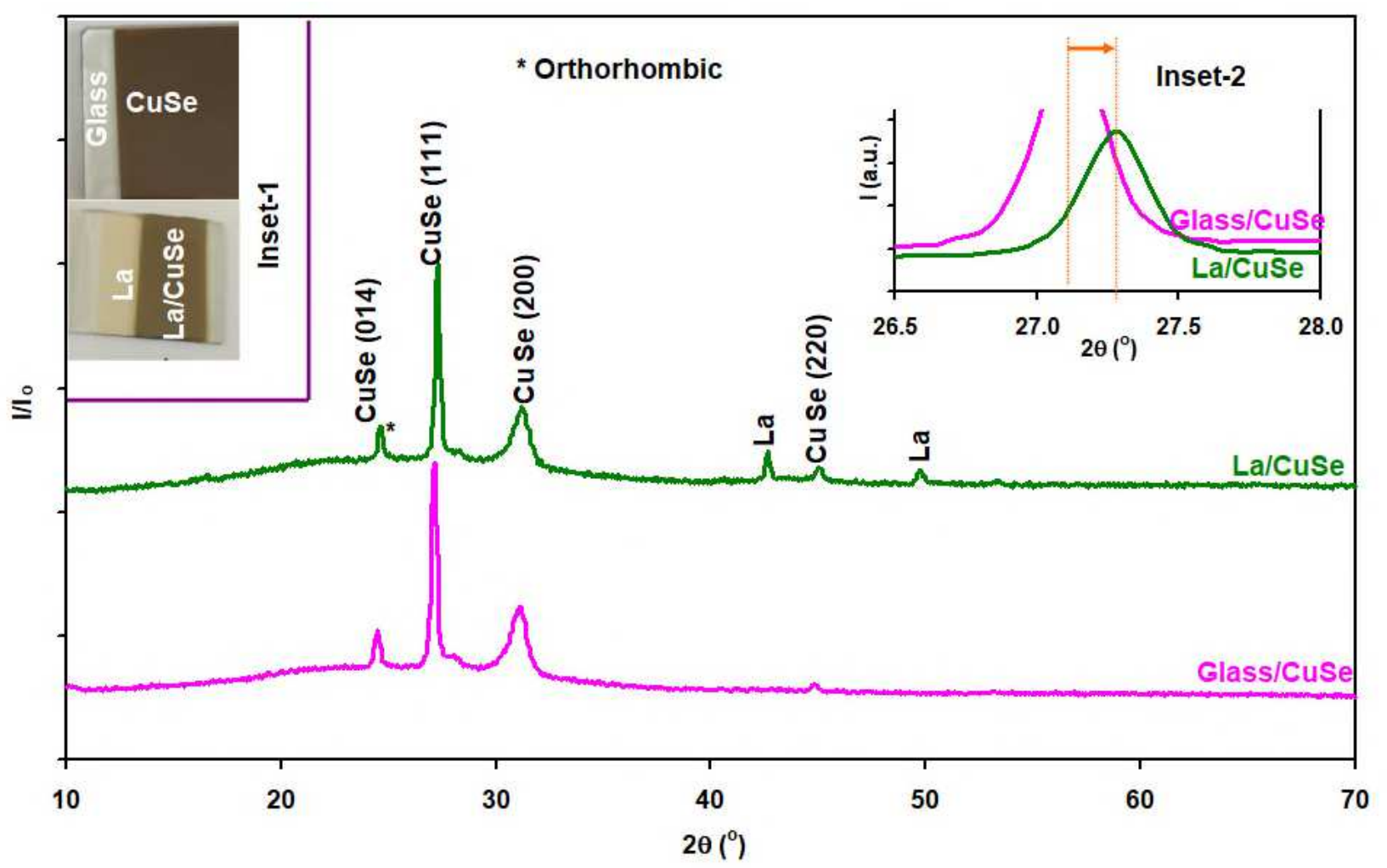

Fig. 1 the X-ray diffraction patterns for glass/CuSe and La/CuSe thin films. Inset-1 shows the optical images for the samples. Inset-2 is an enlargement of the maximum peak. 


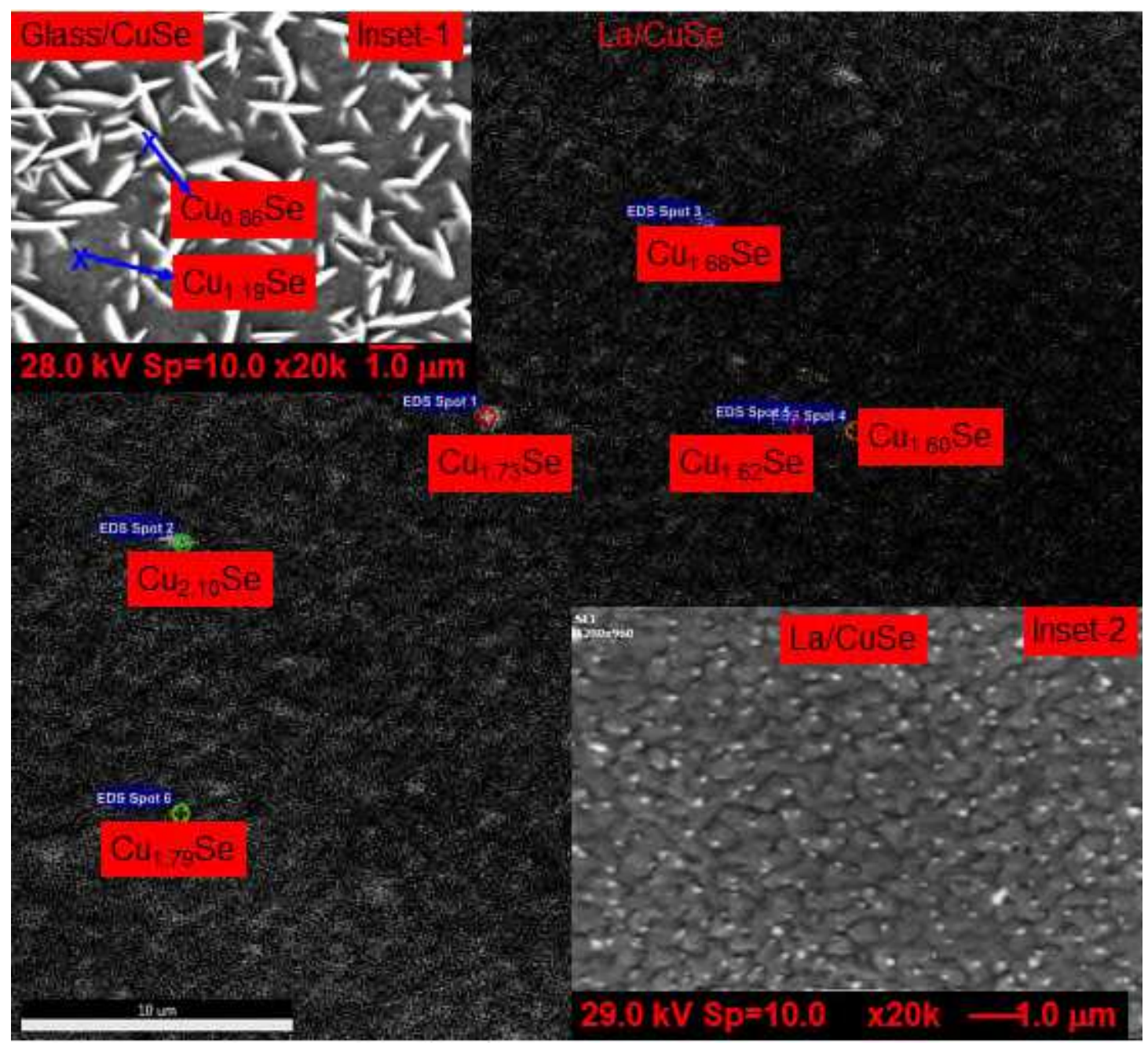

Fig. 2 the scanning electron microscopy images for La/CuSe films. The red boxes in the figure show the energy dispersive X-ray spectral results. Inset -1 and inset-2 show the SEM images for glass/CuSe and $\mathrm{La} / \mathrm{CuSe}$ films, respectively. 

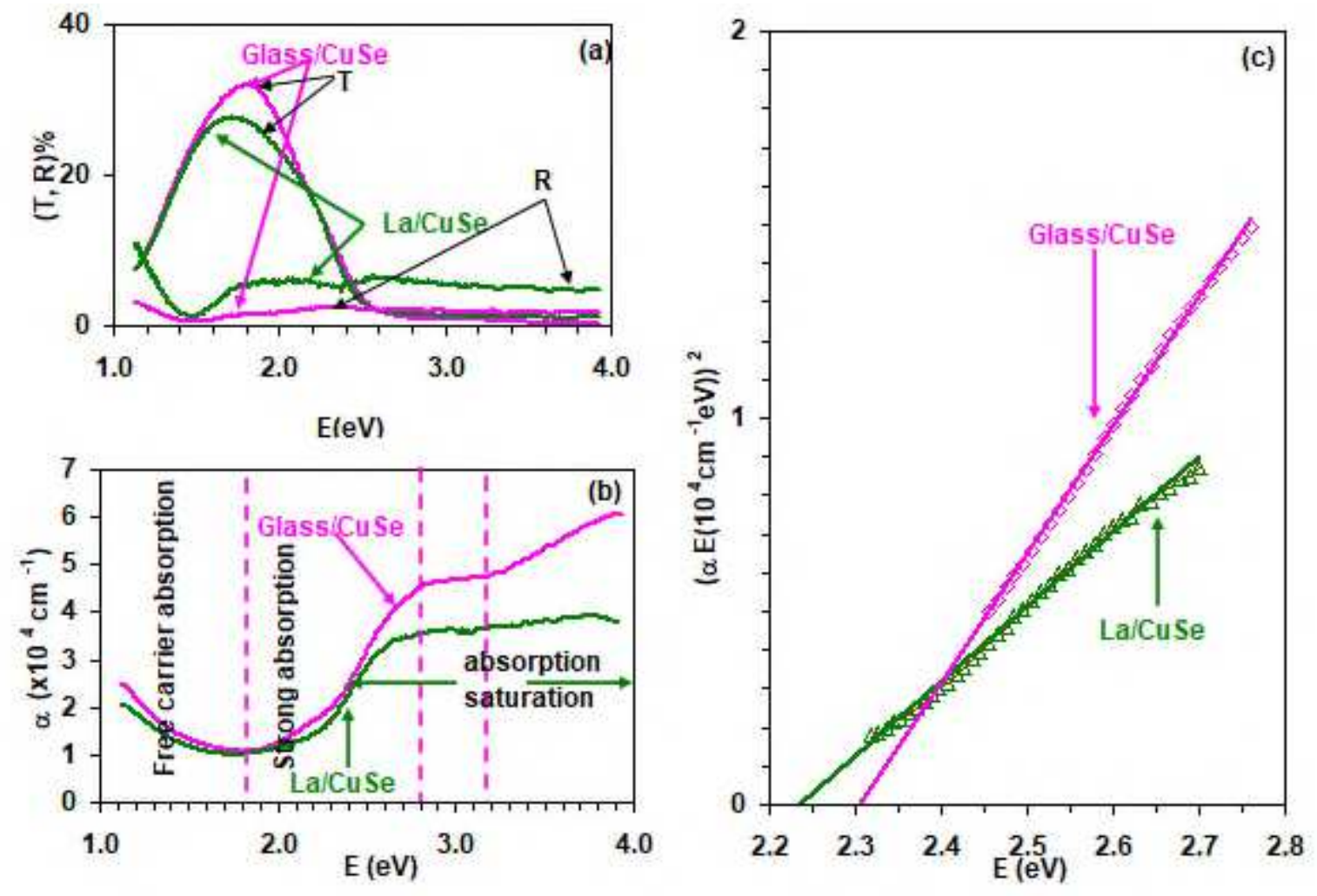

Fig. 3 The optical (a) transmittance and reflectance, (b) absorption coefficient spectra and (c) the Tauc's equation fitting for glass/CuSe and $\mathrm{La} / \mathrm{CuSe}$ films.
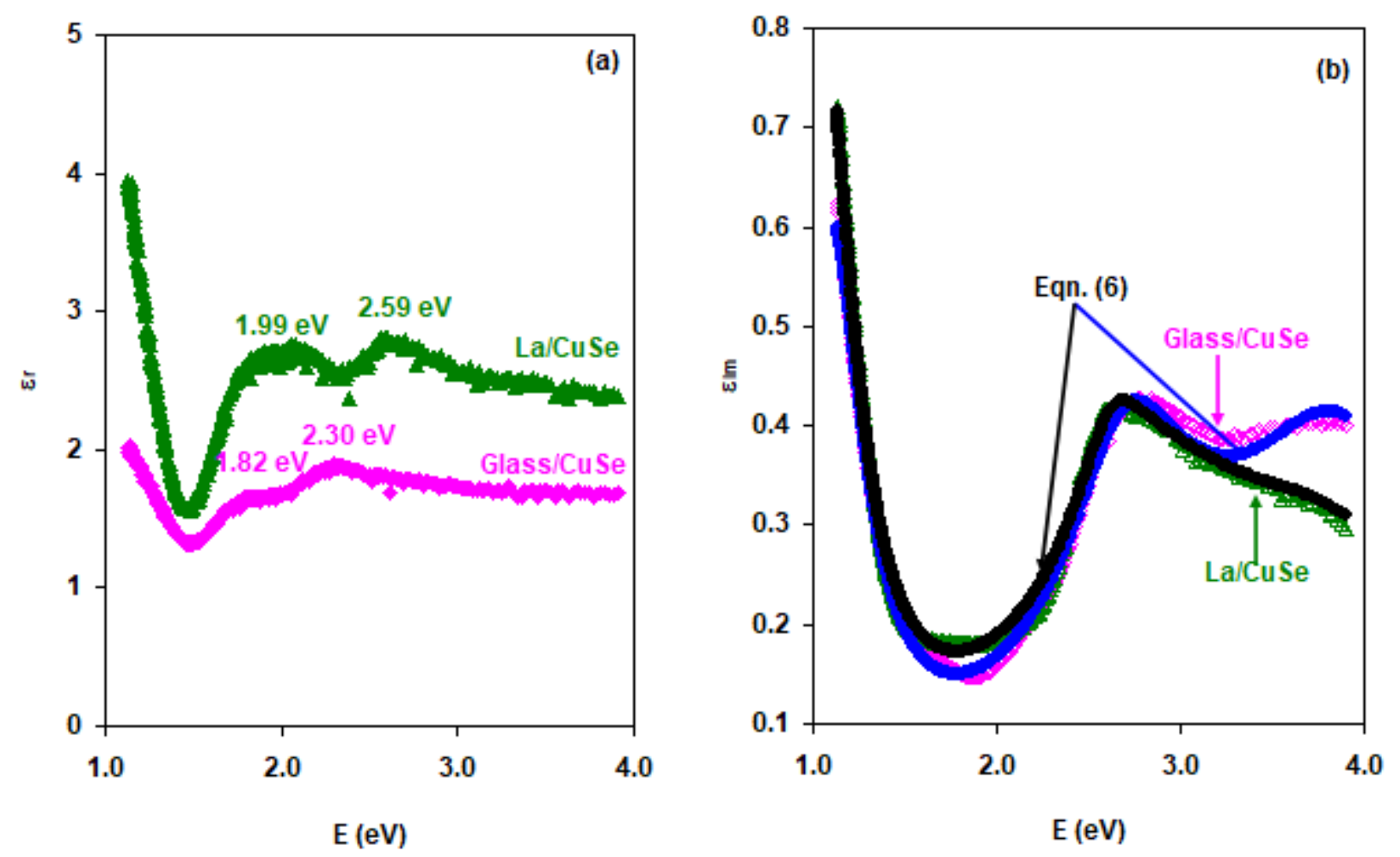

Fig. 4 (a) the $\varepsilon_{r}$ and (b) the $\varepsilon_{i m}$ spectra for the glass/CuSe and $\mathrm{La} / \mathrm{CuSe}$ films. The blue and black circles are the Drude-Lorentz fittings for the imaginary part of dielectric constant. 

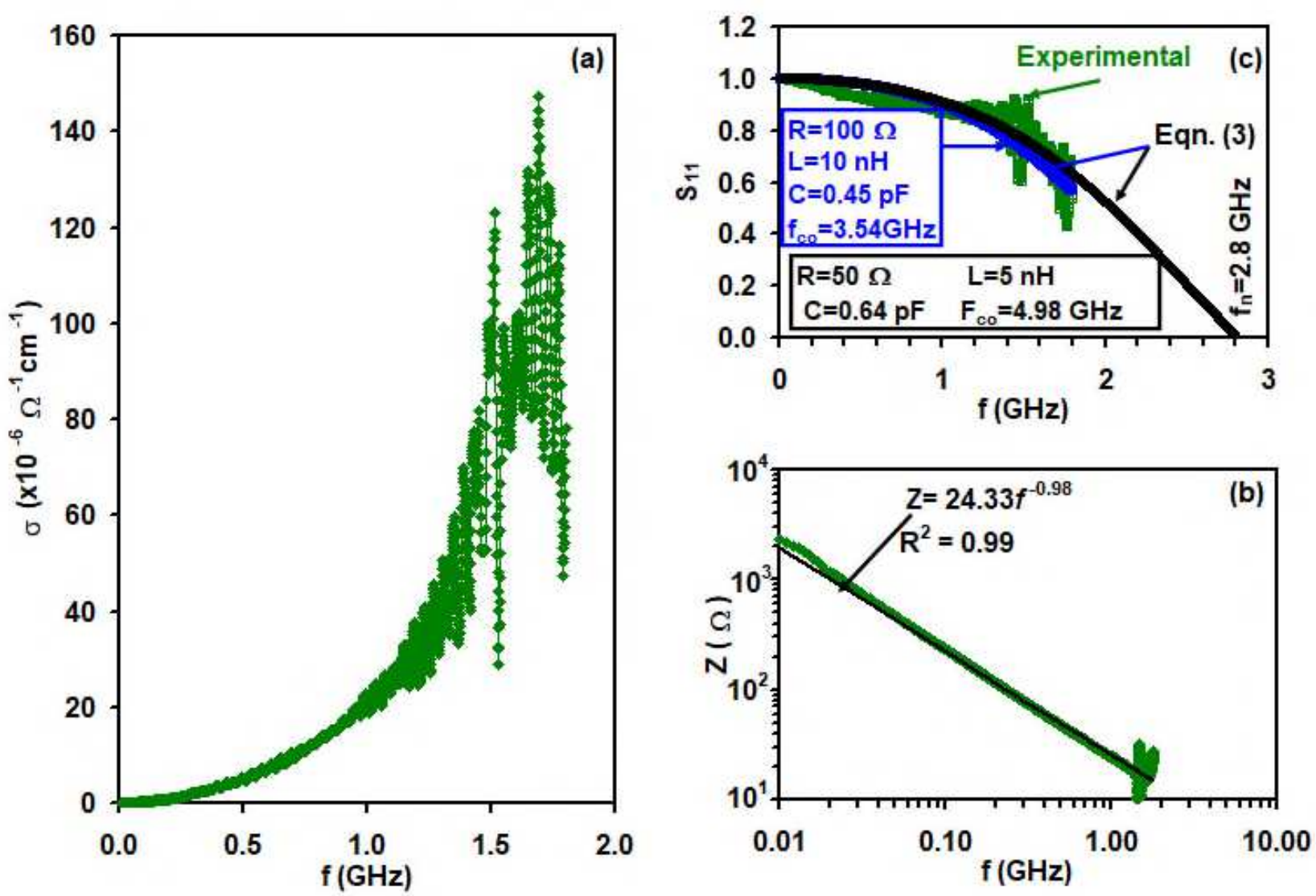

Fig. 5 (a) the conductivity, (b) the impedance and (c) the magnitude of the reflection coefficient spectra for $\mathrm{La} / \mathrm{CuSe} / \mathrm{Ag}$ devices. 